\title{
Analysis of oil \& gas pipelines environmental accidents in China
}

\author{
Li Huiting ${ }^{1,2, a}$, Han Mei ${ }^{1, b}$, Yuan Peng ${ }^{2, c}$, WangHaitao ${ }^{1, d}$ Sun Fei ${ }^{2, e}$ \\ ${ }^{1}$ Department of Urban Water Environmental Research, Chinese Research Academy of \\ Environmental Sciences, Beijing 100012, China \\ ${ }^{2}$ School of Geography and Environment, Shandong Normal University, Jinan Shandong, 250014, \\ China; \\ a2533205571@qq.com, bhanmei568568@126.com, 'cyuanpeng@craes.org.cn, \\ d929600414@qq.com, ${ }^{\mathrm{e}}$ sunfei5218@sina.com
}

\begin{abstract}
Keywords: oil \& gas pipeline; environmental accidents; statistical analysis; countermeasure Abstract: With the rapid development of oil \& gas pipeline construction, the pollution accidents are increasing in quantity. The safe operation of pipelines has aroused widespread concern. This study collected the historical cases of environmental accidents in the domestic oil \& gas pipelines in recent 10 years. The impact of the types of environmental events, the level of events, the causes of events, the influence of different types of environmental receptors and the spatial distribution of accidents of oil \& gas pipelines accidents were analyzed. In order to prevent or reduce the occurrence of pipeline accidents, the paper puts forward the risk prevention and control suggestions based on case analysis: the establishment of the basic database of pipeline data; strengthen the environmental risk assessment and management of pipelines; and strengthen the management of environmental contingency plans.
\end{abstract}

\section{Introduction}

As the fifth transport industry pipeline transportation is widely used in petrochemical industry, energy industry, urban construction and other fields. Pipeline failures are characterized as low-probability, high-consequence events. As the mainly way of the oil and gas transportaion, pipeline occupies an important position in the national economy ${ }^{[1]}$. Since our country established the first crude oil pipeline in Xinjiang in 1958, the gas pipelines has been going through 60 years of development. As the end of 2016, the total mileage of China's long-distance oil and gas pipelines is about $126,000 \mathrm{~km}^{[2]}$. With the construction and development of pipelines, frequent pipeline failures have led to serious environmental pollution and huge economic losses.

Environmental risks of pipelines are prominent. Unlike fixed-source enterprises, once a pipeline ruptures and leaks, materials directly enter the external environment and are prone to fire and explosion, polluting the water environment, causing serious casualties and serious pollution. Therefore, the risk prevention of environmental emergencies in pipeline has a great significance for ensuring environmental safety. This paper analyzes the cases of oil and gas pipeline accidents in our country for nearly ten years to study the law of occurrence and provide a scientific basis andimproves the safety management level.

\section{Current Situation of Environmental Risk Management of Oil and Gas Pipeline in China}

Since the 1980s, China began to pay attention to the environmental protection of oil and gas pipelines. Since the mid-1990s, China began to study the impact of long-distance oil pipeline projects on the ecological environment ${ }^{[3]}$. With the rapid development of pipeline construction, environmental pollution and risk prevention issues are also increasingly prominent. A leak occurred in the underground oil pipeline of PetroChina in Shaanxi provincen 2009, causing Yellow River polliution. Dalian Petroleum International Storage and Transportation corporation occurred pipeline explosion accident. in 2010, resulting in a large number of crude oil leaks and caused a fire, part of the crude oil into the vicinity of the sea, causing some sea pollution. According to relevant data, the average incidence of oil-gas pipeline accidents in China is 3 times / $\left(10^{3} \mathrm{~km} \cdot \mathrm{a}\right)$, which is 6 times of the incidence of pipeline accidents in the United States, 7.5 times in Canada and 8.6 times in Europe ${ }^{[4]}$. 
The traumatic lesson brought about by the pipeline failures sounded the alarm for the safety management of the pipeline. In 2010, China promulgated the "Oil and Gas Pipeline Protection Law". In 2012, industry standard for oil and gas pipeline risk assessment methodology was released, that "Method for Risk Assessment of Oil and Gas Pipelines Part 1: Semi-quantitative Evaluation Method" [5]. In 2017, MEP organized the preparation of "Guideline for Preparing Emergency Plan for Sudden Environmental Incidents in Oil and Gas Pipeline (Draft for Comment)" ${ }^{16]}$. Its purpose is gradually strengthen the prevention and control of oil and gas pipeline safety.

\section{Case Analysis}

There are many causes of pipeline failures, and the consequences are serious. Only in the case of analysis of unexpected environmental accidents of oil and gas pipelines can we continually sum up the experiences and lessons to prevent or mitigate the accident.

A total of 42 cases from 2007 to 2017 were collected in this paper and detailed analysis from the aspects of environmental accident type, accident reason, accident level, impact on different types of environmental receptors and the spatial distribution of accidents. Case sources of information include: MEP notification information, related books ${ }^{[7-8]}$, internet information etc.

\section{pipeline type and event level}

The statistical analysis of 42 cases of oil-gas pipeline failures show that the main types of accident pipelines are oil pipelines, with 32 accidents occurring, accounting for $76 \%$ of the total number of accidents.

Judging from the level of environmental accidents in pipelines, there were 3 major-level incidents, accounting for $7.1 \%$ of the total number. All these accidents caused drinking water pollution; 2 were major incidents, 24 were general incidents and other 13 cases were pending or not scheduled.

\section{Analysis of the cause of the accident}

The cause of pipeline failure can be divided into seven types of destruction by third parties, corrosion, sabotage, improper operation, material / welding / equipment failure, natural disasters and other causes ${ }^{[9]}$. These risk factors will result in leaks, explosions, fires or types of pipeline accidents where either or both are combined. Figure 1 presents the cause of pipeline failures. The major causes of pipeline failures are third-party damage, natural and geological hazards and sabotage. Third-party damage is mainly destroyed by construction.

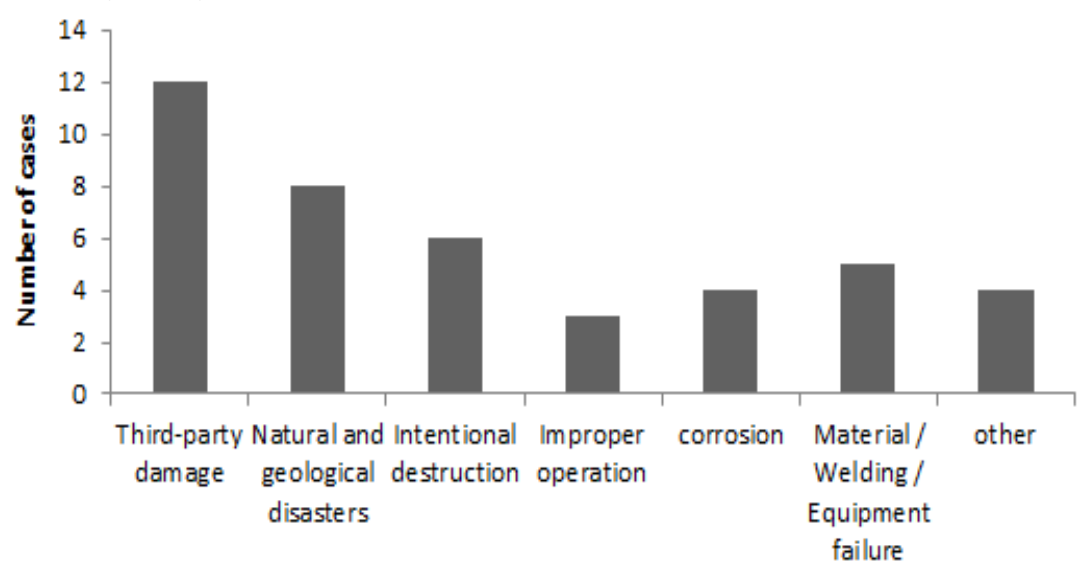

Figure 1 Cause analysis of oil and gas pipeline accident

\section{Accident type analysis}

According to the types of pipeline failures, sudden environmental incidents of oil and gas pipelines can be classified into leaks, fires or explosions caused by leakage, and fire explosions caused by leakage, Figure 2 shows that the pipeline failures are mainly to leakage, a total of 27 cases, accounting for $64.3 \%$ of the total accident, followed by a leak explosion, a total of 3 , accounting for $7.1 \%$. The 
types of gas pipeline accidents were leaks and leak explosions, all of which occurred from 5, accounting for $11.9 \%$ of the total. Among the 27 cases of oil spill accidents, there were 7 cases of leakage caused by third party damage, 6 cases of sabotage, 4 cases of natural and geological disasters, 4 cases of material / construction / equipment failure, and corrosion cause 3 cases, and another 3 cases unknown.

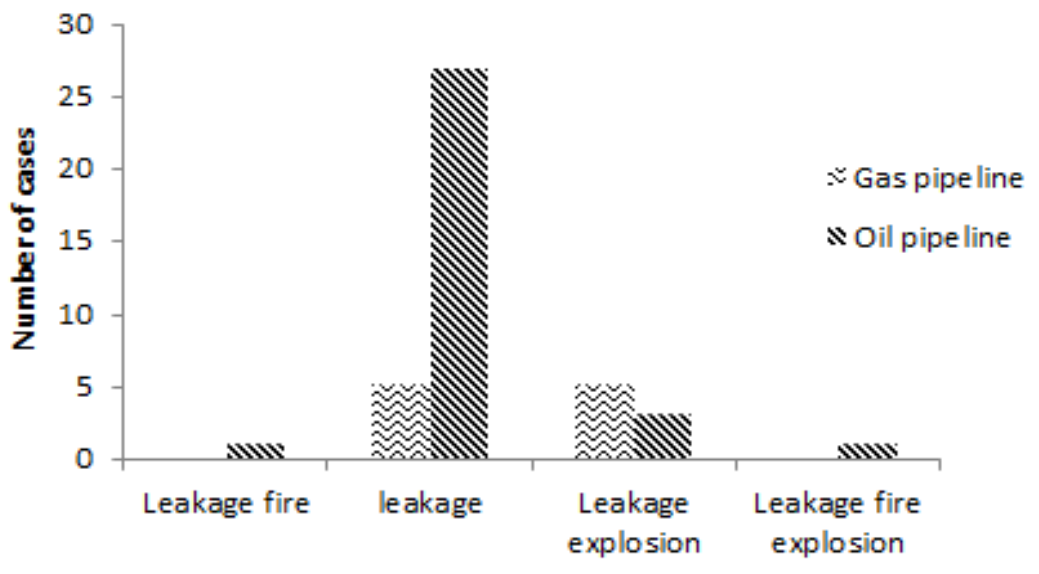

Figure 2 Pipeline failures type classification

\section{Environmental Receptor Type Analysis}

Oil pipeline environmental emergencies may cause site, water and offshore sourced pollution. The types of environmental receptors affected after the accident were analyzed in Figure 3. Among 32 cases of oil pipeline accidents, 17 cases were caused by rivers and reservoirs, 12 cases were contaminated site(including forest land, farmland and mountains), and 3 were contaminated offshore sourced pollution, accounting for $53.2 \%, 37.5 \%$ and $9.3 \%$ of the total respectively.

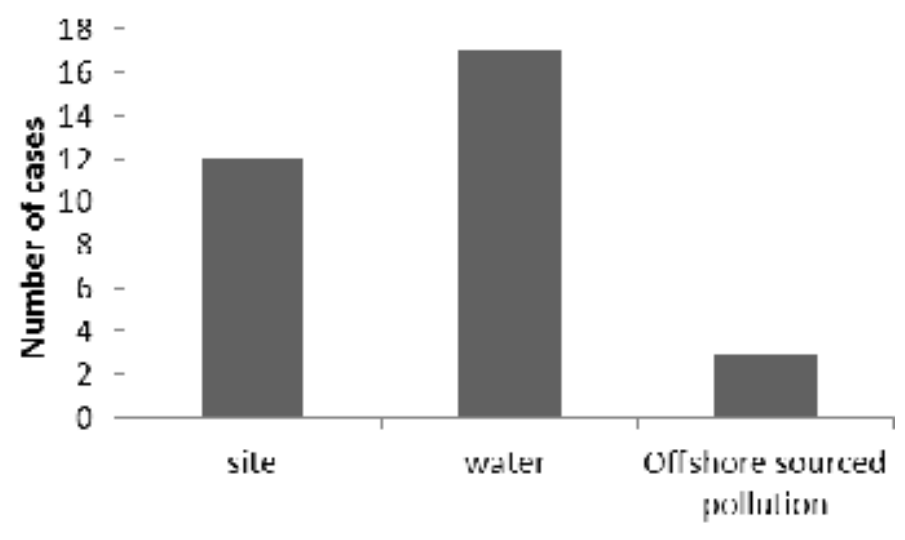

Figure 3 Oil pipeline sudden environmental events receptor type

\section{Spatial Analysis}

From the spatial distribution point of view, the most frequent pipeline failures are Shaanxi Province, from 2007 to 2017, a total of 9 cases occurred, accounting for $21.4 \%$ of the total number of cases; followed by Shandong Province, there are 4; again in Zhejiang Province, Guangdong Province and Liaoning Province, all from 3. 


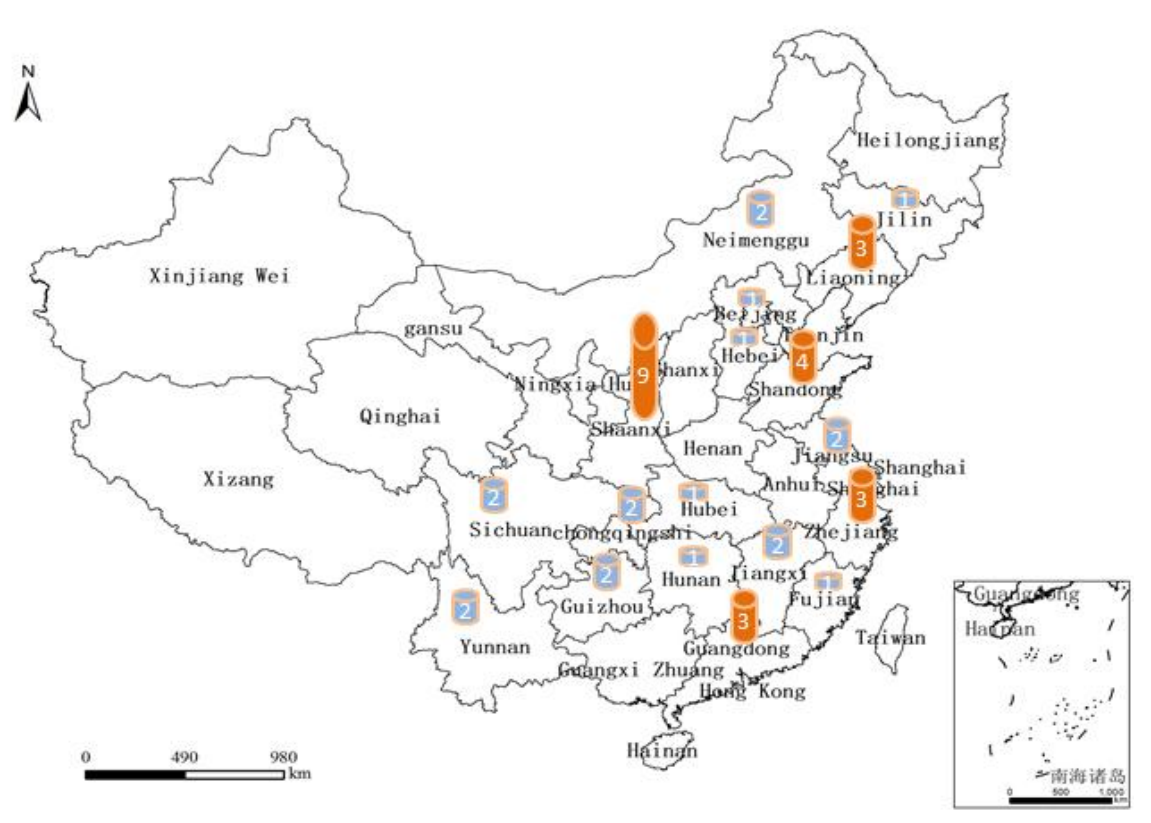

Figure 4 the spatial distribution of accidents

\section{Oil and gas pipeline accident prevention and control recommendations}

According to the analysis of oil and gas pipeline accident,, it can besuggested to focus on the following aspects of work.

\section{Establish a database of pipeline inf rastructure information}

Pipeline basic information system is the basis of pipeline safety management and accident emergency decision-making. The mainly reason of the oil and gas pipeline accidents in China is construction, drawing on the experience of the United States National Pipeline Mapping System, we should speed up the establishment of oil and gas pipeline geographic information management system platform to standardize the pipeline data acquisition objects, scope, data content, data formats. By combined with pipeline accidents management and failure factors we should focus on information on environmental risk receptors in high-risk areas and building GIS-based oil and gas pipeline basic information database to provide data support for emergency response and management.

\section{Strengthen management of oil and gas pipelines environmental risk assessment}

According to the case analysis, the main consequence of accidents in our country is the water pollution. Therefore, when designing the oil pipeline, the pipe should be kept a safe distance from the river as far as possible. The necessary pipelines to cross the river should be provided with buffer zones and emergency pools to prevent the expansion of pollution and protect the water environment. For areas prone to be destroyed by natural geological disasters, shall be set up retaining walls, slope protection and drainage ditches to prevent the pipeline from being destroyed by rain erosion.

\section{Strengthening environmental emergency plan management}

Emergency plan is the respondent in the face of unexpected environmental events, to minimize the unexpected and the damage by the pre-set work program ${ }^{[10]}$. Based on the pipeline environmental risk assessments, according with possible scenarios of oil and gas pipelines accidents, the company will strengthen the management of unexpected environmental incidents in pipelines, conduct training and exercises on a regular basis, master the handling and disposal technologies for unexpected environmental incidents in oil and gas pipelines, understand the use of emergency equipment and clear reserves of emergency supplies. At the same time, it will increase the public's participation in the plan and raise people's awareness of accident risk prevention . 


\section{Acknowledgement}

This research was financially supported by the National Key Technology R\&D Program (2015BAK16B04).

\section{References}

[1] Shuai Jian.Oil and Gas Pipeline Accidents in US and Their Inspiration[J].OGST,2010,29(11): 806-809.

[2] Gao Peng,Tan Zhe,Liu Guangren et all. New progress of China's oil and gas pipeline construction in 2016 [J]. International Petroleum Economy, 2017,25(03):26-33.

[3] Zhao Hanqing. Causes of oil and gas pipeline incidents in China and environmental precautions[J]. Oil \& Gas Storage and Transportation, 2015,34(04):368-372.

[4] Fang Jianping. Case analysis of oil and gas pipeline accidents at home and abroad[J]. Petroleum and chemical equipment,2016,19(09):90-93.

[5] SY/T6891.1-2012, Risk assessment method of oil and gas pipelines first parts: semi-quantitative evaluation method[S].

[6] Ministry of environmental protection. Guidelines for preparing emergency plans for environmental emergencies in oil and gas pipelines (Draft for comments) [S].2017.

[7] Zhang Lijun et all. Typical cases of environmental emergencies (first series) [M].Beijing: China Environment press,2011,48-56.

[8] Zhai Qing et all. Typical cases of environmental emergencies (Second Series) [M].Beijing: China Environment press,2015,24-52.

[9] Di Yan, Shuai Jian, Wang Xiaolin et all. Study on Methods for Classifying Oil \& Gas Pipeline Incidents[J]. Chinese Journal of Safety Science,2013,23(07):109-115.

[10] General Office of the State Council. Measures for the administration of emergency preplan[Z].2013. 